Award Number: W81XWH-08-1-0030

TITLE: Regulation of Prostate Cancer Bone Metastasis by DKK1

PRINCIPAL INVESTIGATOR: Gregory A. Clines, M.D., Ph.D.

CONTRACTING ORGANIZATION: University of Alabama at Birmingham Birmingham, AL 35294-0111

REPORT DATE: April 2012

TYPE OF REPORT: Annual

PREPARED FOR: U.S. Army Medical Research and Materiel Command Fort Detrick, Maryland 21702-5012

DISTRIBUTION STATEMENT:

Approved for public release; distribution unlimited

The views, opinions and/or findings contained in this report are those of the author(s) and should not be construed as an official Department of the Army position, policy or decision unless so designated by other documentation. 


\section{REPORT DOCUMENTATION PAGE}

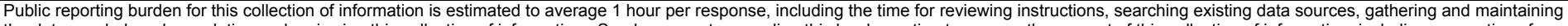

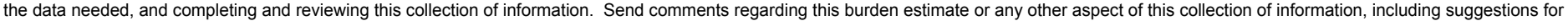

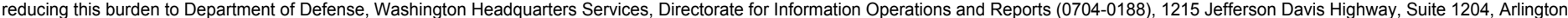

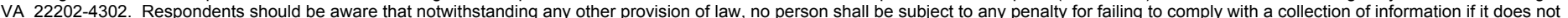
display a currently valid OMB control number. PLEASE DO NOT RETURN YOUR FORM TO THE ABOVE ADDRESS.

\begin{tabular}{l|l|l} 
1. REPORT DATE (DD-MM-YYYY) & 2. REPORT TYPE & 3. DATES COVERED (FrOm - TO)
\end{tabular}

April $2012 \quad$ Annual

1 April 2011 - 28 Feb 2012

4. TITLE AND SUBTITLE

Regulation of Prostate Cancer Bone Metastasis by DKK1

5a. CONTRACT NUMBER

5b. GRANT NUMBER

W81XWH- 0 8-1-0030

5c. PROGRAM ELEMENT NUMBER

6. AUTHOR(S)

Gregory A. Clines, M.D., Ph.D.

5d. PROJECT NUMBER

5e. TASK NUMBER

( Р ПО⿴囗

5f. WORK UNIT NUMBER

\section{PERFORMING ORGANIZATION NAME(S) AND ADDRESS(ES)}

8. PERFORMING ORGANIZATION REPORT NUMBER

University of Alabama at

Birmingham

Birmingham, AL 35294-0111

\section{SPONSORING / MONITORING AGENCY NAME(S) AND ADDRESS(ES)}

U.S. Army Medical Research and Materiel Command

Fort Detrick, Maryland 21702-5012

Fort
10. SPONSOR/MONITOR'S ACRONYM(S)

11. SPONSOR/MONITOR'S REPORT NUMBER(S)

12. DISTRIBUTION / AVAILABILITY STATEMENT

Approved for public release; distribution unlimited

\section{SUPPLEMENTARY NOTES}

\section{ABSTRACT}

Osteoblastic bone metastasis is a common complication of advanced prostate cancer, resulting in pain and pathologic fracture. Dickkopf homolog 1 (DKK1) is a secreted inhibitor of osteoblast Wnt signaling pathway and hypothesized to be a central regulator of prostate cancer osteoblastic bone metastasis. The purpose of this proposal is to examine mechanisms of DKK1 regulation by prostate cancer cells and determine whether DKK1 overexpression in bone blocks the formation of osteoblastic bone lesions in animal models of bone metastasis. We have now shown that human prostate cancer cell lines that produce osteolytic, but not osteoblastic, bone lesions in animal models of bone metastasis express significant amounts of DKK1 and this expression is correlated with the absence of DNA methylation at the DKK1 promoter CpG island. Our preliminary data points to a central role of DKK1 in prostate cancer bone metastasis and expect this work to translate into the development of novel therapeutic targets to treat this malignancy complication.

\section{SUBJECT TERMS}

Prostate cancer, Bone metastasis, Dickkopf homolog 1, Wnt signaling

\begin{tabular}{|c|c|c|c|c|c|}
\hline \multicolumn{3}{|c|}{ 16. SECURITY CLASSIFICATION OF: } & \multirow{2}{*}{$\begin{array}{l}\text { 17. LIMITATION } \\
\text { OF ABSTRACT } \\
\text { UU }\end{array}$} & \multirow[t]{2}{*}{$\begin{array}{l}\text { 18. NUMBER } \\
\text { OF PAGES }\end{array}$} & $\begin{array}{l}\text { 19a. NAME OF RESPONSIBLE PERSON } \\
\text { USAMRMC }\end{array}$ \\
\hline $\begin{array}{l}\text { a. REPORT } \\
\text { U }\end{array}$ & $\begin{array}{l}\text { b. ABSTRACT } \\
\text { U }\end{array}$ & $\begin{array}{l}\text { c. THIS PAGE } \\
\text { U }\end{array}$ & & & $\begin{array}{l}\text { 19b. TELEPHONE NUMBER (include area } \\
\text { code) }\end{array}$ \\
\hline
\end{tabular}




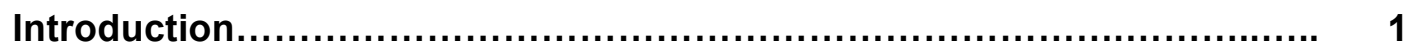

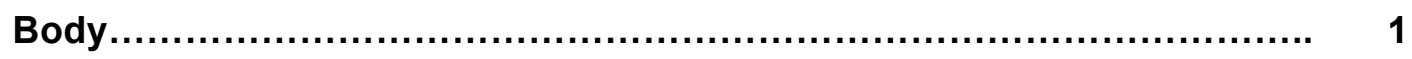

Key Research Accomplishments............................................... $\quad 2$

Reportable Outcomes.............................................................. $\quad 2$

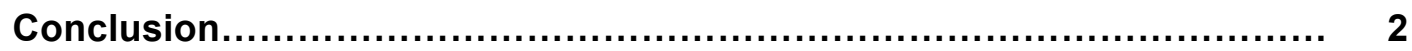

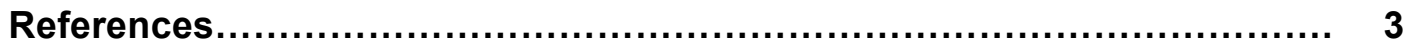

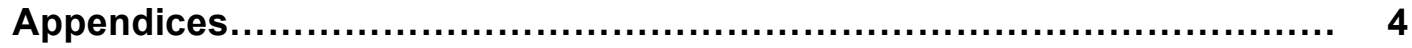

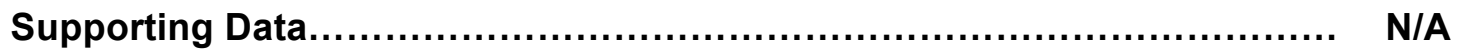




\section{INTRODUCTION}

Osteoblastic bone metastasis is a common complication of advanced prostate cancer, resulting in pain and pathologic fracture (1). In mouse models and human clinical studies of prostate cancer, tumor-produced endothelin-1 (ET-1) activates the osteoblast endothelin A receptor and increases new bone formation (2). In previously published work from our group, we demonstrated that dickkopf homolog 1 (DKK1), a negative canonical Wnt signaling regulator, is reduced by ET-1 resulting in enhanced canonical Wnt signaling activity and new bone formation (3). Others have shown that DKK1 secretion from prostate cancer cells themselves also contribute to bone microenvironment DKK1 (4). We hypothesized that DKK1 is a central regulator of prostate cancer bone metastasis. The purpose of this proposal is to examine mechanisms of DKK1 regulation by prostate cancer cells and determine whether DKK1 overexpression in bone blocks the formation of osteoblastic bone lesions in animal models of bone metastasis. Understanding the role of DKK1 in bone metastasis will facilitate the development of modulators of this factor and other Wnt signaling members. The development of such novel and targeted therapies to bone would represent a significant advancement in the treatment of prostate cancer metastasis to bone.

\section{BODY}

Task 1: Determine if the osteoblastic response to ET-1 is blocked by Dkk1 transgenic overexpression targeted to bone in mouse models of prostate cancer bone metastasis

In Task 1, Dkk1 ${ }^{\mathrm{Ob}}$ mice in the C57BL/6 genetic background were to be bred to C57BL/6 nude animals. However, it remained unclear whether human cancer cells commonly used in mouse metastasis models (BALB/c nude) form bone lesions similarly in the C57BL/6 background. In addition since mice carrying the nude mutation are poor breeders, the SCID mutation $\left(P r k d c^{\text {scid }}\right)$ was a preferred immunodeficient model. A pilot experiment was performed which demonstrated that the tumor take of the LuCaP23.1 xenograft was 9/10 for BALB/c nude mice and $1 / 10$ for C57BL/6 nude mice (Fisher's exact test; $p=0.0011$ ). A similar pilot experiment was preformed using the ARCaPm prostate cancer cells. Tumor take was $6 / 6$ for $\mathrm{BALB} / \mathrm{c}$ nude vs. $1 / 6$ for C57BL/6 SCID. Based on the low tumor appearance in the C57BI/6 backgrounds in both prostate cancer bone metastasis models, mice in the BALB/c background will be used. A modified breeding strategy as reported in last year's report was proposed so that the study mice will possess at least $87.5 \%$ of the BALB/c background to maximize the number of mice that accept tumor. ARCaPm prostate cancer cells were inoculated into the left cardiac ventricle of WT and $D k k 1^{\mathrm{Ob}}$ mice. At 12 weeks of age when radiographic evidence of bone lesions were evident, the mice were euthanized and bones were harvested as detailed in the original proposal. We are currently in the process of performing bone histomorphometry and other measurements on these mice and expect to have this task completed within 3-4 months.

Task 2: Determine how DKK1 production from bone cells and tumor is regulated in vivo in osteoblastic bone metastasis

The work proposed in this task is dependent on Task 1 and will be performed concurrently. We expect to have this Task completed within 4-6 months.

Task 3: Determine if $\mathbf{D k k 1}$ is inactivated by promoter CpG island hypermethylation in prostate cancer 
Task 3 has been completed and data encompassing this task has been submitted to the journal Experimental and Clinical Metastasis. This manuscript is attached as an appendix to this report. The conclusions from this work are the following:

1. Prostate cancer cell lines that produce osteoblastic/mixed lesions in animal models of bone metastasis secrete more DKK1 than the PC3 osteolytic cancer cell line.

2. DKK1 promoter methylation correlates with DKK1 expression.

3. Demethylation restores DKK1 expression osteoblastic/mixed prostate cancer cell lines.

4. In the PC3 prostate cancer cell line with large DKK1 expression, Wnt signaling is unexpectedly active.

5. Kremen, the DKK1 receptor, is downregulated in PC3 cells suggesting a mechanism for DKK1 resistance.

6. Overexpression of Kremen in the PC3 prostate cancer cell line partially restores Wnt inhibition by DKK1.

A portion of Task 3 proposed to examine DKK1 methylation in human prostate cancer bone metastasis samples. We received bone metastasis frozen sections from Dr. Robert Vessella, a collaborator on this grant. Unfortunately, the amount of DNA harvested from these samples was inadequate for methylation-specific sequencing using the bisulfite conversion strategy. We now know that significantly more DNA is required for this strategy than was available from the samples provided.

\section{KEY RESEARCH ACCOMPLISHMENTS}

- Osteolytic, but not osteoblastic, prostate cancer cells express DKK1

- Osteolytic, but not osteoblastic, prostate cancer cells have unmethylated DKK1 promoter

- Demethylation corrects DKK1 suppression

- Low Kremen (DKK1 receptor) blocks Wnt suppression from high DKK1 in PC3 prostate cancer cells

- Kremen overexpression in PC3 prostate cancer cells partially restores DKK1 inhibition of canonical Wnt signaling.

\section{REPORTABLE OUTCOMES}

The manuscript entitled "DKK1 and Kremen Expression Predicts the Osteoblastic Response to Bone Metastasis" has been submitted to Clinical and Experimental Metastasis. This manuscript has been reviewed and revisions are currently in process

\section{CONCLUSION}

Bone metastasis is a significant complication of advanced prostate cancer that causes pain and pathologic fracture. This work is aimed at uncovering the role of DKK1 in prostate cancer bone metastasis. We have discovered a correlation between behavior of prostate cancer in 
bone, DKK1 expression and DNA methylation of the DKK1 promoter. Studies that are in progress will examine whether overexpression of DKK1 in the bone microenvironment blocks bone metastasis in an animal model. Medical and social costs of bone metastasis are high. This work is expected to translate into improved treatments for prostate cancer bone metastasis and facilitate the development of therapeutic targets to DKK1.

\section{REFERENCES}

1. G. A. Clines, T. A. Guise, Endocrine-Related Cancer 12, 549 (2005).

2. J. J. Yin et al., Proceedings of the National Academy of Sciences of the United States of America 100, 10954 (2003).

3. G. A. Clines et al., Molecular Endocrinology 22, 486 (2007).

4. C. L. Hall, A. Bafico, J. Dai, S. A. Aaronson, E. T. Keller, Cancer Research 65, 7554 (2005).

\section{APPENDICES}

The manuscript entitled "DKK1 and Kremen Expression Predicts the Osteoblastic Response to Bone Metastasis" submitted to Clinical and Experimental Metastasis. 


\begin{tabular}{|c|c|}
\hline \multicolumn{2}{|l|}{ Manuscript Number: } \\
\hline Full Title: & DKK1 and Kremen Expression Predicts the Osteoblastic Response to Bone Metastasis \\
\hline Article Type: & Original Research \\
\hline Keywords: & Bone metastasis; DKK1; osteoblast; Kremen; Wnt signaling \\
\hline Corresponding Author: & $\begin{array}{l}\text { Gregory A Clines, M.D., Ph.D. } \\
\text { University of Alabama at Birmingham } \\
\text { Birmingham, Alabama UNITED STATES }\end{array}$ \\
\hline \multicolumn{2}{|l|}{$\begin{array}{l}\text { Corresponding Author Secondary } \\
\text { Information: }\end{array}$} \\
\hline Corresponding Author's Institution: & University of Alabama at Birmingham \\
\hline \multicolumn{2}{|l|}{$\begin{array}{l}\text { Corresponding Author's Secondary } \\
\text { Institution: }\end{array}$} \\
\hline First Author: & Katrina L Clines, M.S. \\
\hline \multicolumn{2}{|l|}{ First Author Secondary Information: } \\
\hline \multirow[t]{2}{*}{ Order of Authors: } & Katrina L Clines, M.S. \\
\hline & Gregory A Clines, M.D., Ph.D. \\
\hline \multicolumn{2}{|c|}{ Order of Authors Secondary Information: } \\
\hline Abstract: & $\begin{array}{l}\text { Bone metastasis is a common complication of advanced breast and prostate cancer. } \\
\text { Tumor-secreted Dickkopf homolog } 1 \text { (DKK1), an inhibitor of canonical Wnt signaling, } \\
\text { dictates the osteoblast response to the invading cancer. The aims of this study were to } \\
\text { determine if DKK1 expression predicts how bone responds to metastasis and to } \\
\text { discover mechanisms that regulate cancer DKK1 expression in bone. DKK1 } \\
\text { expression was examined in a panel of breast and prostate cancer cell lines and a } \\
\text { prostate cancer xenograft. DKK1 expression was highest in MDA-MB-231 and PC3 } \\
\text { cells that yield osteolytic lesions. LnCaP, C4-2, C4-2B, LuCaP23.1, T47D, ZR-75-1, } \\
\text { MCF-7, ARCaP and ARCaPM cancer cells that generate osteoblastic, mixed or no } \\
\text { bone lesions exhibited lower DKK1 expression. Epigenetic regulation of DKK1 had } \\
\text { been previously reported in other cancers and was investigated as a mechanism of } \\
\text { DKK1 downregulation. Methylation-specific sequencing revealed DKK1 promoter } \\
\text { methylation of the LnCaP, C4-2, C4-2B and T47D cancer cell lines, resulting in } \\
\text { transcriptional repression. In cells with absent DKK1 promoter methylation, Wnt } \\
\text { signaling activity was analyzed since this signaling pathway itself regulates DKK1 } \\
\text { expression. Wnt signaling was in fact active in the cells examined, including MDA-MB- } \\
231 \text { and PC3 cancer cells that secrete sufficient DKK1 expected to block canonical } \\
\text { Wnt signaling. A mechanism of DKK1 resistance observed in the osteolytic cell lines } \\
\text { was at least partially due to downregulation of the DKK1 receptors Kremen1 and } \\
\text { Kremen2. In conclusion, combined DKK1 and Kremen expression in cancer cells may } \\
\text { serve as predictive markers and therapeutic targets in bone metastasis. }\end{array}$ \\
\hline
\end{tabular}




\title{
DKK1 and Kremen Expression Predicts the Osteoblastic Response to Bone Metastasis
}

\author{
Katrina L. Clines ${ }^{1}$ and Gregory A. Clines ${ }^{1,2}$ \\ ${ }^{1}$ Department of Medicine, Division of Endocrinology, Diabetes and Metabolism, \\ University of Alabama at Birmingham, Birmingham, Alabama and \\ ${ }^{2}$ Veterans Affairs Medical Center, Birmingham, Alabama
}

\section{Corresponding Author:}

Gregory A. Clines, M.D., Ph.D.

Assistant Professor of Medicine and Cell Biology

Division of Endocrinology, Diabetes, and Metabolism

Department of Medicine

University of Alabama at Birmingham

Endocrinology Section, Birmingham VA Medical Center

1808 7th Avenue South, BDB 730

Birmingham, AL 35294-0012

Office: (205) 934-4187

Fax: (205) 975-9372

Email: clines@uab.edu 


\begin{abstract}
Bone metastasis is a common complication of advanced breast and prostate cancer. Tumorsecreted Dickkopf homolog 1 (DKK1), an inhibitor of canonical Wnt signaling, dictates the osteoblast response to the invading cancer. The aims of this study were to determine if DKK1 expression predicts how bone responds to metastasis and to discover mechanisms that regulate cancer DKK1 expression in bone. DKK1 expression was examined in a panel of breast and prostate cancer cell lines and a prostate cancer xenograft. DKK1 expression was highest in MDA-MB-231 and PC3 cells that yield osteolytic lesions. LnCaP, C4-2, C4-2B, LuCaP23.1, T47D, ZR-75-1, MCF-7, ARCaP and $\mathrm{ARCaP}_{\mathrm{M}}$ cancer cells that generate osteoblastic, mixed or no bone lesions exhibited lower DKK1 expression. Epigenetic regulation of DKK1 had been previously reported in other cancers and was investigated as a mechanism of DKK1 downregulation. Methylation-specific sequencing revealed DKK1 promoter methylation of the LnCaP, C4-2, C4-2B and T47D cancer cell lines, resulting in transcriptional repression. In cells with absent DKK1 promoter methylation, Wnt signaling activity was analyzed since this signaling pathway itself regulates DKK1 expression. Wnt signaling was in fact active in the cells examined, including MDA-MB-231 and PC3 cancer cells that secrete sufficient DKK1 expected to block canonical Wnt signaling. A mechanism of DKK1 resistance observed in the osteolytic cell lines was at least partially due to downregulation of the DKK1 receptors Kremen1 and Kremen2. In conclusion, combined DKK1 and Kremen expression in cancer cells may serve as predictive markers and therapeutic targets in bone metastasis.
\end{abstract}


Keywords: Bone metastasis, DKK1, osteoblast, Kremen, Wnt signaling

\author{
Abbreviations \\ DKK1 Dickkopf homolog 1 \\ ELISA Enzyme-linked immunosorbant assay \\ ET-1 Endothelin-1 \\ ETAR Endothelin A receptor \\ HER2 Human epidermal growth factor receptor 2 \\ LDL Low-density lipoprotein \\ MESD Mesoderm development candidate 2 protein \\ MSX2 Msh homeobox 2 \\ SOST Gene encoding sclerostin protein \\ TCF T-cell transcription factor \\ Wnt Wingless/integrated
}




\section{Introduction}

Bone metastasis is a common complication of advanced prostate and breast cancer and defines a point in the disease when cure is unlikely. The invasion of tumor cells into bone irrevocably alters the bone microenvironment and initiates a skeletal response that is dependent on the type of tumor [1]. Breast cancer bone metastasis typically results in massive osteolysis from the secretion of osteoclast-activating factors, such as parathyroid hormone-related protein and others [2]. Prostate cancer classically forms osteoblastic lesions under the direction of osteoblast-activating factors that include endothelin-1 (ET-1), Wnt signaling proteins and bone morphogenetic proteins [3, 4]. Both osteolytic and osteoblastic bone metastases represent heightened states of bone turnover but differ in the extent to which osteoblast bone formation or osteoclast bone resorption predominates.

Dickkopf homolog 1 (DKK1) is a secreted inhibitor of canonical Wnt signaling that may predict cancer cell behavior in bone. During normal bone homeostasis, DKK1 is secreted from mature osteoblasts that then feeds-back to inhibit Wnt signaling of osteoblast precursors [5]. DKK1 operates by sequestering the LDL-related proteins 5 and 6 co-receptors from the $G$ protein-coupled protein receptor Frizzled and thus blocks Wnt signaling activation [6]. Negative feedback of DKK1 supports tight control of bone formation and thus prevents excessive osteoblast activity. This role of DKK1 is illustrated by the osteopenic phenotype with DKK1 transgenic overexpression in mice $[7,8]$.

DKK1 regulates the osteoblastic response to invading cancer cells in bone and therefore influences the balance between bone formation and resorption [5, 9]. This idea was first proposed when DKK1 was identified as a causal factor in osteoblast suppression characteristic of multiple myeloma bone disease [10]. Since this first report, DKK1 has been implicated in other forms of cancer and bone metastasis. In animal models of prostate cancer bone metastasis, DKK1 overexpression in the prostate cancer cell line C4-2B, which normally forms 
mixed osteoblastic-osteolytic bone lesions, resulted in the formation of primarily osteolytic lesions [11]. Conversely, knockdown of DKK1 expression in the PC3 prostate cancer cell line resulted in increased osteoblastic potential [11].

Cancer cells not only secrete DKK1 but also are able to manipulate the secretion of DKK1 from the osteoblast. This is mediated by tumor-secreted ET-1, which activates the osteoblast endothelin A receptor (ETAR) and downregulates osteoblast DKK1 [12]. ET-1 therefore promotes pathologic bone formation by ensuring DKK1 is quelled, permitting excessive osteoblast activity and bone formation. ETAR antagonists slow progression of osteoblastic lesions in animal models of osteoblastic bone metastasis as well in human clinical trials, which suggests an important role of DKK1 in bone metastasis [3,13,14]. Collectively, DKK1 secreted by both cancer cells and mature osteoblasts contribute to bone microenvironment DKK1, and influences osteoblast development and pathologic bone formation in bone metastasis.

We set out to examine the extent to which DKK1 expression in breast and cancer cell lines predicts behavior in bone. In a panel of breast and prostate cancer cell lines, DKK1 expression correlated with the degree of osteoblast suppression characteristic of an osteolytic skeletal phenotype. Both epigenetic methylation of the DKK1 promoter and transcriptional mechanisms were found to regulate DKK1. In the osteolytic cell lines that secreted the most DKK1, Wnt signaling was unexpectedly found to be active. We provide evidence that active Wnt signaling reported in these aggressive osteolytic cell lines is maintained by a mechanism of DKK1 resistance. 


\section{Materials and Methods}

Reagents:

The prostate cancer cell lines LnCaP and PC3, the breast cancer cell lines T47D, ZR-75-1 and MCF-7, and the colon cancer cell line COLO205 were obtained from ATCC (Manassas, VA). The ARCaP and $\mathrm{ARCaP}_{\mathrm{M}}$ prostate cell lines were obtained from Novicure Biotechnology (Birmingham, AL). Dr. Leland Chung, Cedars-Sinai Medical Center, provided the prostate cancer cell lines C4-2 and C4-2B. Dr. Robert Vessella, University of Washington, provided the LuCaP23.1 prostate cancer xenograft. The breast cancer cell line MDA-MB-231 utilized in these studies is a bone avid subline obtained from the parental MDA-MB-231 cell line [15]. 5aza-2'-deoxycytidine was obtained from Sigma-Aldrich (St. Louis, MO).

\section{Messenger RNA expression analysis:}

Messenger RNA expression was determined by real-time RT PCR using an iScript SYBR Green RT-PCR kit (Bio-Rad, Hercules, CA) and a MylQ Single-Color Real-Time PCR Detection System (Bio-Rad, Hercules, CA). The following primers were utilized: DKK1-F: tagcaccttggatgggtatt, DKK1-R: atcctgaggcacagtctgat, LRP5-F: gttcggtctgacgcagtaca, LRP5-R: gtccatcacgaagtccaggt, LRP6-F: cccatgcacctggttctact, LRP6-R: ccaagccacagggatacagt, SOSTF: ggaaagtccagggactggtt, SOST-R: catctacagttgcccccagt, MESD-F: agctgggaggtgcttcagta, MESD-R: cagcagggactatgcagtga, KRM1-F: atccagatggagacgtgagc, KRM1-R: tccttgtagcagccaaggtt, KRM2-F: acacctgagatgctgtgctg, KRM2-R: ttcctgtccgactttggtc. Relative differences in mRNA concentration were determined by subtracting the Ct (threshold cycle) of the study gene from the Ct of the housekeeping gene RPL32 (F: cagggttcgtagaagattcaaggg and R: cttggaggaaacattgtgagcgatc) $\left(\Delta=\mathrm{Ct}_{\text {gene }}-\mathrm{Ct}_{\mathrm{RPL32}}\right)$. The mean of the lowest DKK1 expressing cell line $\left(\Delta_{\text {low }}\right)$ was subtracted from each of the cell lines $\left(\Delta_{\text {sample }}\right)$; $\left(\right.$ mean $\Delta_{\text {low }}-\Delta_{\text {sample }}$ $=\varepsilon)$. The fold difference was calculated as $2^{\varepsilon}$. 


\section{DKK1 ELISA}

DKK1 protein in cancer cell conditioned media was determined by ELISA (R\&D Systems, Minneapolis, MN). After collection of media, the cells were trypsinized and counted.

\section{Methylation-specific sequencing:}

Genomic DNA was isolated from the selected cancer cell lines and prostate cancer xenograft. The DKK1 CpG island and flanking DNA were PCR amplified to produce a $449 \mathrm{bp}$ fragment (F: aggggtgaagagtgtcaaagg; $\mathrm{R}$ : aggttcttgatagcgttgga). The fragment was sequenced to confirm the published sequence. Isolated DNA was then subjected to bisulfite treatment using an EZ DNA Methylation-Direct kit (Zymo Research Corporation, Irvine, CA). The bisulfitetreated DNA was PCR amplified using published primers (F: ggggtgaagagtgttaaagg; R: aaaccatcatctcaaaaaaactcaa) that flank the DKK1 CpG island to produce a 326 bp fragment [16]. Amplified fragments were sequenced. The presence of a cytosine indicated that the base was protected by methylation.

Immunohistochemistry:

Cancer cells were grown on collagen-coated glass cover slips and fixed for 30 minutes in 4\% paraformaldehyde/1\% Triton X-100. Samples were washed and incubated with $0.3 \%$ hydrogen peroxide for 30 minutes and washed in PBS. Cells were blocked with $1 \%$ BSA (Vector Laboratories), PBS washed and incubated for 30 minutes with a mouse anti- $\beta$-catenin antibody (Millipore, Billerica, MA) at a 1:500 dilution. Slides were washed with PBS and incubated with Alexa 488 goat anti-rabbit (1:400 dilution) (Invitrogen) for 30 minutes. Cells were washed with PBS/0.1\% Triton X-100. Samples were nuclear counterstained with 300 nM 4',6diamidino-2-phenylindole (DAPI) for 5 minutes. 


\section{Wnt reporter assay}

Cells were transfected with TOPFlash or FOPFlash Wnt reported vectors (Millipore, Billerica, MA) plus Renilla luciferase as a normalization standard using Lipofectamine 2000 transfection reagent (Life Technologies, Grand Island, NY). Forty-eight hours after transfection, DualLuciferase Reporter Assays (Promega, Madison, WI) were performed using a BioTek Synergy 2 microplate reader (BioTek, Winooski, VT). The construction of the dominant-negative TCF3 construct has been described (Wong, et al, J Cell Biol, 2003). A human Kremen1 cDNA vector clone was obtained from ATCC (Manassas, VA). The cDNA was then subcloned into the pCMV-Sport6 expression plasmid (Life Technologies, Grand Island, NY).

\section{Statistical analyses}

Statistical analyses were performed using Prism 4.00 software. Comparisons of two groups were performed using an unpaired, two-tailed $t$ test. Significant differences are indicated $\left(^{*}=\right.$ $\left.p<0.05 ;{ }^{* *}=p<0.01 ;{ }^{* \star *}=p<0.001\right)$.

\section{Results}

\section{Cancer cell DKK1 expression predicts bone phenotype}

DKK1 mRNA expression and protein secretion into the surrounding medium was surveyed in selected breast and prostate cancer cells lines and correlated with the phenotypic response of the cancer cells in bone (Fig. 1). The androgen-dependent prostate cancer cell line LNCaP and the subline derivatives C4-2 and C4-2B [17] expressed nearly undetectable DKK1. Of these, only C4-2B elicits a skeletal response after inoculation with mixed osteoblastic and osteoclastic characteristics. The human prostate cancer xenograft LuCaP23.1 produces osteoblastic bone lesions with intratibial inoculation [18], and little DKK1 expression was 
detected. The prostate cancer cell line ARCaP and the bone avid osteoblastic subline $\mathrm{ARCaP}_{\mathrm{M}}$ [17] expressed more $\mathrm{DKK} 1 . \mathrm{ARCaP}_{\mathrm{M}}$ is a reliable model of osteoblastic bone metastasis. The breast cancer cell lines T47D, ZR-75-1 and MCF-7 also produce osteoblastic lesions in animal models [3] and expressed detectable amounts of DKK1. The breast cancer cell line MDA-MB231 [2] and the prostate cancer cell line PC3 [19] elicit strong osteolytic responses in animal models of bone metastasis. These two cell lines expressed the highest amounts of DKK1. Among the cancer cells lines tested, mRNA concentration correlated well with absolute protein secreted into the surrounding medium (Fig. 1).

\section{Methylation of the DKK1 CpG island downregulates DKK1 expression}

The cancer cell lines selected for study demonstrated wide variation in DKK1 expression. Alteration in DNA methylation pattern is one mechanism that promotes tumorigenesis. The DKK1 gene contains a 233 bp CpG island surrounding the transcriptional start site and a portion of the first exon [16] (Fig. 2). This area has a GC content of $68 \%$ containing 18 potential cytosine methylation points where a cytosine precedes a guanine. This $\mathrm{CpG}$ island is a focus of methylation previously reported to regulate DKK1 transcription in colon cancer [16, 20], acute myeloid leukemia [21], malignant glioma [22] and multiple myeloma [23]. The extent to which a similar epigenetic mechanism of gene regulation regulates DKK1 expression in prostate and breast cancer cells was tested. Using a methylation-specific sequencing approach, the cell lines with negligible expression (LNCaP, C4-2, C4-2B and T47D) had some degree of methylation of the $\mathrm{CpG}$ island indicating a putative mechanism of transcriptional repression (Fig. 2). Methylation of the DKK1 CpG island was not detected in the remaining cell lines.

The impact of DNA methylation on DKK1 transcriptional repression was examined in C4-2B and T47D cells, the two cell lines with detectable DKK1 CpG island methylation that reliably produce osteoblastic lesions in animal models. The unmethylated PC3 and MDA-MB-231 cells lines served as controls. Treatment with the DNA demethylating agent 5-aza-2'-deoxycytidine 
resulted in a nearly 500-fold increase in DKK1 mRNA in the C4-2B cell line, strongly indicating that methylation is responsible for transcriptional repression (Fig. 3). Demethylation did not significantly alter DKK1 mRNA concentration in the T47D breast cancer cell line suggesting that likely other unrecognized transcriptional mechanisms cooperate in the downregulation of DKK1 expression. Interestingly, both MDA-MB231 and PC3 cancer cell lines exhibited a marginal increase in DKK1 mRNA despite being unmethylated at the DKK1 promoter. This result was likely due to indirect phenomena, such as the presence of epigenetically controlled transcriptional activators that regulate DKK1 transcription.

\section{Regulation of DKK1 expression by Wnt signaling}

The absence of DKK1 promoter methylation within the LuCaP23.1, ARCaP, ARCaP, ZR75-1, MCF-7, MDA-MB-231 and PC3 cells indicated that other mechanisms regulated DKK1 expression. Wnt signaling itself is one candidate. DKK1 expression is regulated, at least partly, by TCF/LEF Wnt signaling responsive elements located within the DKK1 promoter, and thus fits with DKK1 operating in a negative feedback loop regulating Wnt signaling [24]. A unifying mechanism of DKK1 regulation by Wnt signaling in the studied cancer cell lines was investigated by assessing the degree of nuclear localization of $\beta$-catenin, a marker for active Wnt signaling. The cell lines that reproducibly form bone lesions in animal models $(\mathrm{C} 4-2 \mathrm{~B}$, T47D, ZR-75-1, MCF-7, MDA-MB-231 and PC3) were selected for examination and all demonstrated nuclear $\beta$-catenin staining (Fig. 4A). Controls using secondary antibody without primary antibody showed no staining (data not shown). C4-2B, T47D, ZR-75-1 and MCF-7 cell lines showed additional staining of the cell membrane. This staining pattern may indicate $\beta$ catenin reserve and a lower level of Wnt signaling, and/or the presence of maturity adherens junction complexes that associate with $\beta$-catenin. 
A puzzling aspect of the data is that DKK1 itself is a potent inhibitor of Wnt signaling. In most cells, $10-50 \mathrm{ng} / \mathrm{ml}$ of DKK1 is sufficient to block Wnt signaling $[10,12,25]$. DKK1 was once again assayed from conditioned media of the cells that underwent immunofluorescent analysis (Fig. 4A). MDA-MB-231 and PC3 cells secreted more than sufficient quantities of DKK1 (>50 ng/ml) to block Wnt signaling. To confirm active Wnt signaling in these cell line, a dominant-negative expression construct for the mutant form of TCF3 lacking the $\beta$-catenin binding site was co-transfected along with Wnt signaling reporter vectors into the MDA-MD-231 and PC3 cell lines. The strategy efficiently downregulated Wnt signaling, again suggesting that Wnt signaling is active in these cells (Fig. 4B). These data support that MDA-MB-231 and PC3 cell lines are insensitive to the Wnt-suppressive actions of DKK1.

\section{Kremen downregulation causes DKK1 resistance}

DKK1 action is dependent on other Wnt signaling components and dysregulation of these members could result in DKK1 resistance. DKK1 binds to the high-affinity transmembrane Kremen1 and Kremen2 receptors. The DKK1-Kremen receptor complex sequesters LRP5 and LRP6 away from the Wnt ligand and Frizzled receptor, leading to LRP removal from the cell membrane and downregulation of Wnt signaling [26, 27]. Downregulation of LRPs and/or Kremen receptors could render DKK1 inactive. Two other Wnt inhibitors, Sclerostin (encoded by the gene SOST) and mesoderm development candidate 2 protein (MESD), compete with DKK1 for LRP binding [28, 29]. Excessive expression of these Wnt antagonists could mask DKK1-mediated Wnt inhibition. Expression of these genes was assessed in the seven cell lines that produce bone lesions in animal models of bone metastasis (Fig. 5). A consistent pattern of low Kremen1 and Kremen2 expression in MDA-MB-231 and PC3 cells suggested a mechanism of DKK1 resistance.

To test the extent to which expression of Kremen could restore DKK1-mediated Wnt signaling inhibition, Kremen1 was overexpressed in MDA-MB-231 and PC3 cells and Wnt 
signaling was assessed. Kremen1 reduced Wnt signaling in these cancer cell lines suggesting that downregulation of Kremen membrane receptors is in part responsible for DKK1 resistance (Fig. 6).

\section{Discussion}

Risk prediction tools have greatly assisted clinicians in selecting the most effective therapies for patients at the highest risk for cancer progression and metastasis, and in avoiding unnecessary treatments for low risk patients. The Gleason grading system combined with clinical stage and serum prostate-specific antigen have improved prognostic accuracy in prostate cancer patients. Expression of HER2 and the receptors for estrogen and progesterone in conjunction with clinical stage provides prognostic information used to select appropriate treatments for women with breast cancer. Despite these important advances in risk prediction, strategies that identify which patients will develop metastasis to specific organs are currently lacking.

DKK1 may be an ideal marker to predict bone metastasis in patients with early malignancies. DKK1 expression was reported higher in women with hormone-resistant breast cancers, which are more likely to be aggressive and metastasize [30]. Similarly, serum DKK1 was higher in women with breast cancer compared to normal subjects, and in women with breast cancer bone metastasis compared to women with breast cancer metastasis to non-bone sites [20, 31]. Although potentially a valuable bone metastasis marker, DKK1 may be an even better predictor of how the skeleton responds to the invading cancer cells. We now report that DKK1 secretion was the highest in the cell lines that produce osteolytic lesions in animal models of bone metastasis. DKK1 excess would therefore suppress Wnt-mediated osteoblast differentiation and allow uncoupled and unrestricted osteolytic bone resorption. In the case of 
osteoblastic bone metastasis, less DKK1 in the bone microenvironment would permit maximal canonical Wnt signaling in the osteoblast.

The range of DKK1 expression in the cancer cell lines tested was extreme, from high expressing PC3 cells to undetectable DKK1 in C4-2 cells. Epigenetic control of DKK1 had been reported in other malignancies and we examined whether a similar mode of regulation occurred, especially in the breast and prostate cell lines with the very lowest expression. Using a methylation-specific sequencing approach, DNA methylation of the DKK1 CpG island was detected in LnCaP and the cell lines C4-2 and C4-2B derived from parental LnCaP cells. This result is consistent with a previous report in which DKK1 was one of 813 genes methylated in LnCaP cells [32]. This same report also showed that DKK1 was selectively methylated in metastatic prostate cancer, but not in primary prostate cancer, benign tissue adjacent to prostate cancer, or normal prostate. Whether restoration of DKK1 expression using systemic DNA demethylases would reduce prostate cancer burden or possibly convert bone metastases to a more osteolytic phenotype is unclear and merits future study. Progressive decrease in DKK1 expression from the primary tumor to bone metastasis has been reported and may in fact involve dynamic changes in DKK1 CpG island methylation [33]. The only breast cancer cell line methylated at the DKK1 promoter was T47D, which translated into negligible expression. In breast cancers, DKK1 epigenetic inactivation appears to be a less common event and was reported to occur in $19 \%$ of primary tumors analyzed [34]. In the cancer cell lines not methylated at the DKK1 CpG and not subject to epigenetic control, other unidentified mechanisms regulating DKK1 exist. The heat shock family member DNAJB6 is certainly a candidate, and was reported to regulate DKK1 expression in breast cancer cells [35]. The homeobox protein MSX2 regulated DKK1 expression in mesenchymal cells but it is unclear whether this occurs in tumors [36].

Cancer cells with ample DKK1 expression alter the bone microenvironment and regulate osteoblast canonical Wnt signaling. DKK1 also regulates Wnt signaling of the cancer cells 
themselves. In the cancer cell lines with low to negligible DKK1 expression, canonical Wnt signaling would be determined by the balance between Wnt ligand activators and other repressors. Canonical Wnt signaling as measured by $\beta$-catenin nuclear staining was in fact active in these cells. Paradoxically, canonical Wnt signaling was detected in MDA-MB-231 and PC3 cells that produce large amounts of DKK1. One explanation is that MDA-MB-231 and PC3 cells may secrete a biologically inactive protein. However, other groups have reported that these cell lines in fact secrete an active DKK1 protein [11, 37-39]. Resistance to the actions of DKK1 in MDA-MB-231 and PC3 was then proposed. Of the components required for DKK1 action, the Kremen1 and Kremen2 were found to be consistently downregulated in these cell lines. Kremen proteins are high-affinity DKK1 receptors that cooperate with DKK1 to increase the clearance of LRP co-receptors $[26,27]$, resulting in Wnt signaling downregulation. Kremen participates in regulating bone homeostasis and osteoblast biology [40,41]. Overexpression of Kremen1 in MDA-MB-231 and PC3 cancer cells partially rescued the ability of DKK1 to reduce Wnt signaling suggesting a mechanism of DKK1 resistance.

We propose a central mechanism that revolves around DKK1 and Kremen, in which canonical Wnt signaling is independently regulated in cancer cells and osteoblasts in bone metastasis. In breast cancer, Wnt signaling is clearly required for breast cancer cell proliferation and migration $[42,43]$. In breast cancer bone metastasis, TGF- $\beta$ released from the bone matrix during osteolysis is a critical event that supports breast cancer growth in bone [44]. Massive osteolysis characterized by uncoupled bone turnover is made possible through the osteoblast suppressive effects of tumor-secreted DKK1. It is therefore essential for breast cancer cells to possess DKK1 resistance. An analogous mechanism can be applied to PC3 animal models of prostate cancer osteolysis. In the situation of osteoblastic bone metastasis, especially in the case of prostate cancer, active canonical Wnt signaling promotes the malignant potential [45, 46]. Low or even absent DKK1 in the bone microenvironment, regardless of tumor Kremen 
expression, permits active canonical Wnt signaling of the tumor cells and the osteoblasts. Kremen therefore may act as a molecular switch that cooperates with DKK1 to determine how cancer cells behave in bone. Defining expression of these two Wnt regulators may have predictive value when assessing risk for progression in primary tumors and in determining the risk for bone metastasis. DKK1 and Kremen receptors also represent novel therapeutic targets for bone metastasis.

\section{Acknowledgments}

We thank Monica Lewis for technical assistance, and Drs. Theresa Guise and John Chirgwin for helpful discussions. Dr. Barry Gumbiner, University of Virginia, kindly provided the dominantnegative TCF3 construct. This publication was made possible by grants PC073756 from the Department of Defense Prostate Cancer Research Program (GAC) and CA118428 from $\mathrm{NCI} / \mathrm{NIH}(\mathrm{GAC})$. 


\section{References}

1. Weilbaecher KN, Guise TA, McCauley LK (2011) Cancer to bone: a fatal attraction. Nat Rev Cancer 11: 411-25

2. Guise TA, et al. (1996) Evidence for a causal role of parathyroid hormone-related protein in the pathogenesis of human breast cancer-mediated osteolysis. Journal of Clinical Investigation 98: 1544-9

3. Yin JJ, et al. (2003) A causal role for endothelin-1 in the pathogenesis of osteoblastic bone metastases. Proceedings of the National Academy of Sciences of the United States of America 100: 10954-9

4. Dai J, et al. (2008) Prostate cancer induces bone metastasis through Wnt-induced bone morphogenetic protein-dependent and independent mechanisms. Cancer Res 68: 5785-94

5. Pinzone JJ, et al. (2009) The role of Dickkopf-1 in bone development, homeostasis, and disease. Blood 113: 517-25

6. Mao B, et al. (2001) LDL-receptor-related protein 6 is a receptor for Dickkopf proteins. Nature 411: $321-5$

7. Li J, et al. (2006) Dkk1-mediated inhibition of Wnt signaling in bone results in osteopenia. Bone 39: 754-66

8. Guo J, et al. (2010) Suppression of Wnt signaling by Dkk1 attenuates PTH-mediated stromal cell response and new bone formation. Cell Metab 11: 161-71

9. Glinka A, et al. (1998) Dickkopf-1 is a member of a new family of secreted proteins and functions in head induction. Nature 391: 357-62

10. Tian E, et al. (2003) The role of the Wnt-signaling antagonist DKK1 in the development of osteolytic lesions in multiple myeloma. New England Journal of Medicine 349: 2483-94

11. Hall CL, et al. (2005) Prostate cancer cells promote osteoblastic bone metastases through Wnts. Cancer Research 65: 7554-60 
12. Clines GA, et al. (2007) Dickkopf homolog 1 mediates endothelin-1-stimulated new bone formation. Molecular Endocrinology 22: 486-98

13. Drake JM, Danke JR, Henry MD (2010) Bone-specific growth inhibition of prostate cancer metastasis by atrasentan. Cancer Biol Ther 9: 607-14

14. Nelson JB (2005) Endothelin receptor antagonists. World Journal of Urology 23: 19-27

15. Yoneda T, et al. (2001) A bone-seeking clone exhibits different biological properties from the MDA-MB-231 parental human breast cancer cells and a brain-seeking clone in vivo and in vitro. Journal of Bone \& Mineral Research 16: 1486-95

16. Aguilera O, et al. (2006) Epigenetic inactivation of the Wnt antagonist DICKKOPF-1 (DKK1) gene in human colorectal cancer. Oncogene 25: 4116-21

17. Zhau HE, Li CL, Chung LW (2000) Establishment of human prostate carcinoma skeletal metastasis models. Cancer 88: 2995-3001

18. Ellis WJ, et al. (1996) Characterization of a novel androgen-sensitive, prostate-specific antigen-producing prostatic carcinoma xenograft: LuCaP 23. Clin Cancer Res 2: 1039-48

19. Soos G, et al. (1997) Comparative intraosseal growth of human prostate cancer cell lines LNCaP and PC-3 in the nude mouse. Anticancer Res 17: 4253-8

20. Sato $\mathrm{H}$, et al. (2007) Frequent epigenetic inactivation of DICKKOPF family genes in human gastrointestinal tumors. Carcinogenesis 28: 2459-66

21. Suzuki R, et al. (2007) Preferential hypermethylation of the Dickkopf-1 promoter in corebinding factor leukaemia. Br J Haematol 138: 624-31

22. Gotze S, et al. (2010) Frequent promoter hypermethylation of Wnt pathway inhibitor genes in malignant astrocytic gliomas. Int J Cancer 126: 2584-93

23. Kocemba KA, et al. (2012) Transcriptional Silencing of the Wnt-Antagonist DKK1 by Promoter Methylation Is Associated with Enhanced Wnt Signaling in Advanced Multiple Myeloma. PloS ONE 7: e30359 
24. Niida A, et al. (2004) DKK1, a negative regulator of Wnt signaling, is a target of the betacatenin/TCF pathway. Oncogene 23: 8520-6

25. Semenov MV, et al. (2001) Head inducer Dickkopf-1 is a ligand for Wnt coreceptor LRP6. Curr Biol 11: 951-61

26. Mao B, et al. (2002) Kremen proteins are Dickkopf receptors that regulate Wnt/betacatenin signalling. Nature 417: 664-7

27. Mao B, Niehrs C (2003) Kremen2 modulates Dickkopf2 activity during Wnt/LRP6 signaling. Gene 302: 179-83

28. Li X, et al. (2005) Sclerostin binds to LRP5/6 and antagonizes canonical Wnt signaling. J Biol Chem 280: 19883-7

29. Lu W, et al. (2010) Mesd is a universal inhibitor of Wnt coreceptors LRP5 and LRP6 and blocks Wnt/beta-catenin signaling in cancer cells. Biochemistry 49: 4635-43

30. Forget MA, et al. (2007) The Wnt pathway regulator DKK1 is preferentially expressed in hormone-resistant breast tumours and in some common cancer types. Br J Cancer 96: $646-53$

31. Voorzanger-Rousselot N, et al. (2007) Increased Dickkopf-1 expression in breast cancer bone metastases. Br J Cancer 97: 964-70

32. Kim JH, et al. (2011) Deep sequencing reveals distinct patterns of DNA methylation in prostate cancer. Genome Res 21: 1028-41

33. Hall CL, et al. (2008) Dickkopf-1 expression increases early in prostate cancer development and decreases during progression from primary tumor to metastasis.

Prostate 68: 1396-404

34. Suzuki H, et al. (2008) Frequent epigenetic inactivation of Wnt antagonist genes in breast cancer. Br J Cancer 98: 1147-56

35. Mitra A, et al. (2010) DNAJB6 induces degradation of beta-catenin and causes partial reversal of mesenchymal phenotype. J Biol Chem 285: 24686-94 
36. Shao JS, et al. (2005) Msx2 promotes cardiovascular calcification by activating paracrine Wnt signals. J Clin Invest 115: 1210-20

37. Li ZG, et al. (2008) Low-density lipoprotein receptor-related protein 5 (LRP5) mediates the prostate cancer-induced formation of new bone. Oncogene 27: 596-603

38. Bu G, et al. (2008) Breast cancer-derived Dickkopf1 inhibits osteoblast differentiation and osteoprotegerin expression: implication for breast cancer osteolytic bone metastases. Int J Cancer 123: 1034-42

39. Fillmore RA, et al. (2009) Nmi (N-Myc interactor) inhibits Wnt/beta-catenin signaling and retards tumor growth. Int J Cancer 125: 556-64

40. Ellwanger K, et al. (2008) Targeted disruption of the Wnt regulator Kremen induces limb defects and high bone density. Mol Cell Biol 28: 4875-82

41. Schulze J, et al. (2010) Negative regulation of bone formation by the transmembrane Wnt antagonist Kremen-2. PLoS One 5: e10309

42. Liu CC, et al. (2010) LRP6 overexpression defines a class of breast cancer subtype and is a target for therapy. Proc Natl Acad Sci U S A 107: 5136-41

43. Many AM, Brown AM (2010) Mammary stem cells and cancer: roles of Wnt signaling in plain view. Breast Cancer Res 12: 313

44. Yin JJ, et al. (1999) TGF-beta signaling blockade inhibits PTHrP secretion by breast cancer cells and bone metastases development. Journal of Clinical Investigation 103: 197206

45. Robinson DR, Zylstra CR, Williams BO (2008) Wnt signaling and prostate cancer. Current drug targets 9: $571-80$

46. Bisson I, Prowse DM (2009) WNT signaling regulates self-renewal and differentiation of prostate cancer cells with stem cell characteristics. Cell Res 19: 683-97 


\section{Figure Legends}

Fig. 1. DKK1 mRNA and protein expression in cancer cells correlates with bone response. DKK1 mRNA was measured in a panel of breast (T47D, ZR-75-1, MCF-7 and MDA-MB-231) and prostate cancer (C4-2B, C4-2, $\mathrm{LnCaP}, \mathrm{ARCaP}_{\mathrm{M}}, \mathrm{ARCaP}$ and PC3) cell lines and a prostate cancer xenograft (LuCaP23.1). In the cell lines, DKK1 secreted into the surrounding medium was also measured by ELISA and normalized to cell number. Absolute DKK1 values in cell lines with the lowest expression are reported. Closed bars represent cancer cells that produce osteoblastic, mixed or no bone lesions in animal models of bone metastasis. Open bars represent cancer cells that produce osteolytic lesion in animal models of bone metastasis.

Fig. 2. DKK1 methylation patterns in cancer cells. A $233 \mathrm{bp} \mathrm{CpG} \mathrm{island} \mathrm{encompasses} \mathrm{the}$ transcriptional start site and a portion of the first exon of DKK1. Eighteen potential methylation sites were identified within this $\mathrm{CpG}$ island. Methylation-specific sequencing was performed to identify the methyl-deoxycytidines of this CpG island. The COLO205 colon cancer cell line served as a control for methylation as this region of the DKK1 gene was reported methylated [16].

Fig. 3. Demethylation restored DKK1 expression in C4-2B prostate cancer cells. Cancer cell lines were treated with and without $10 \mu \mathrm{M}$ of 5-aza-2'-deoxycytidine (5-dAza-C) for four days. Messenger RNA was isolated from the cells and subjected to DKK1 real-time RT PCR. (NS = not significant; $\left.{ }^{*}=p<0.05 ;{ }^{* *}=p<0.001\right)$.

Fig. 4. Canonical Wnt signaling in cancer cell lines. (A) Seven cancer cell lines utilized in animal models of bone metastasis were analyzed for cellular location of $\beta$-catenin using fluorescence immunostaining. The cells were counterstained with DAPI to identify the nucleus. 
All cells had some degree of nuclear $\beta$-catenin accumulation. Conditioned media collected from this experiment was analyzed for DKK1 by ELISA. (B) MDA-MB-231 and PC3 cells were transfected with a dominant-negative TCF3 (dnTCF) or empty vector plasmids, along with Wnt reporter vectors. The ratio of TOPFlash to FOPFlash luciferase activity (TOP:FOP) indicated relative Wnt signaling activity. $\left({ }^{* *}=p<0.01 ;{ }^{* \star *}=p<0.001\right)$.

Fig. 5. Expression of DKK1 binding partners and Wnt signaling inhibitors. Real-time RT PCR was performed in seven cancer cell lines that produce bone lesions in animal models of bone metastasis. Messenger RNA was analyzed for the genes that encode for LDL-related receptor proteins 5 and 6 (LRP5, LRP6), Sclerostin (SOST), mesoderm development candidate 2 protein (MESD), Kremen1 (Krm1) and Kremen2 (Krm2).

Fig. 6. Overexpression of Kremen1 reduces Wnt signaling. MDA-MB-231 and PC3 cells were transfected with a Kremen1 expression construct or empty vector control, along with Wnt reporter vectors. The ratio of TOPFlash to FOPFlash luciferase activity (TOP:FOP) indicated relative Wnt signaling activity. $\left({ }^{*}=p<0.05 ;{ }^{* *}=p<0.01\right)$. 


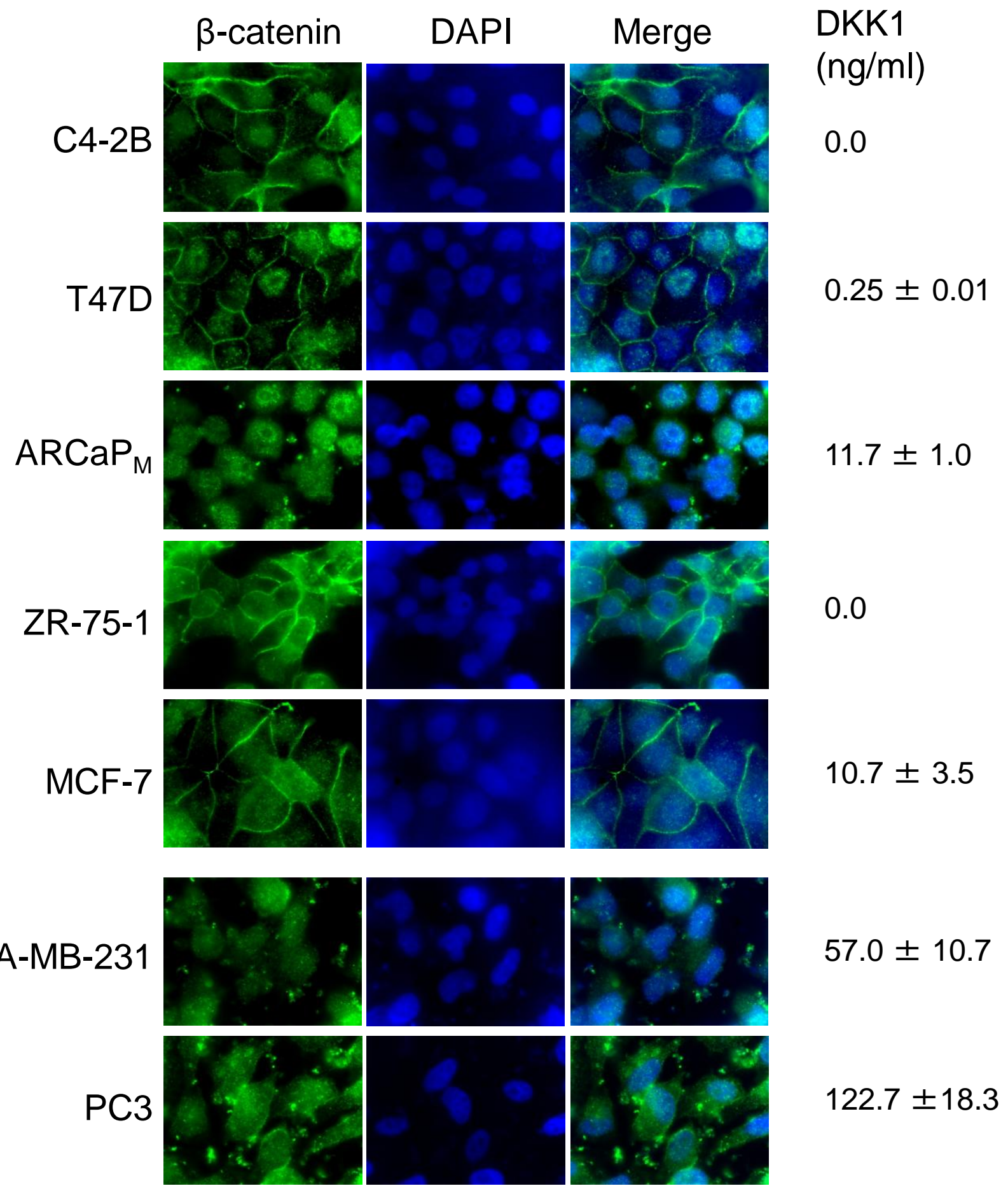

Figure 4A 
Fig. 1

Click here to download line figure: Figure 1.ppt
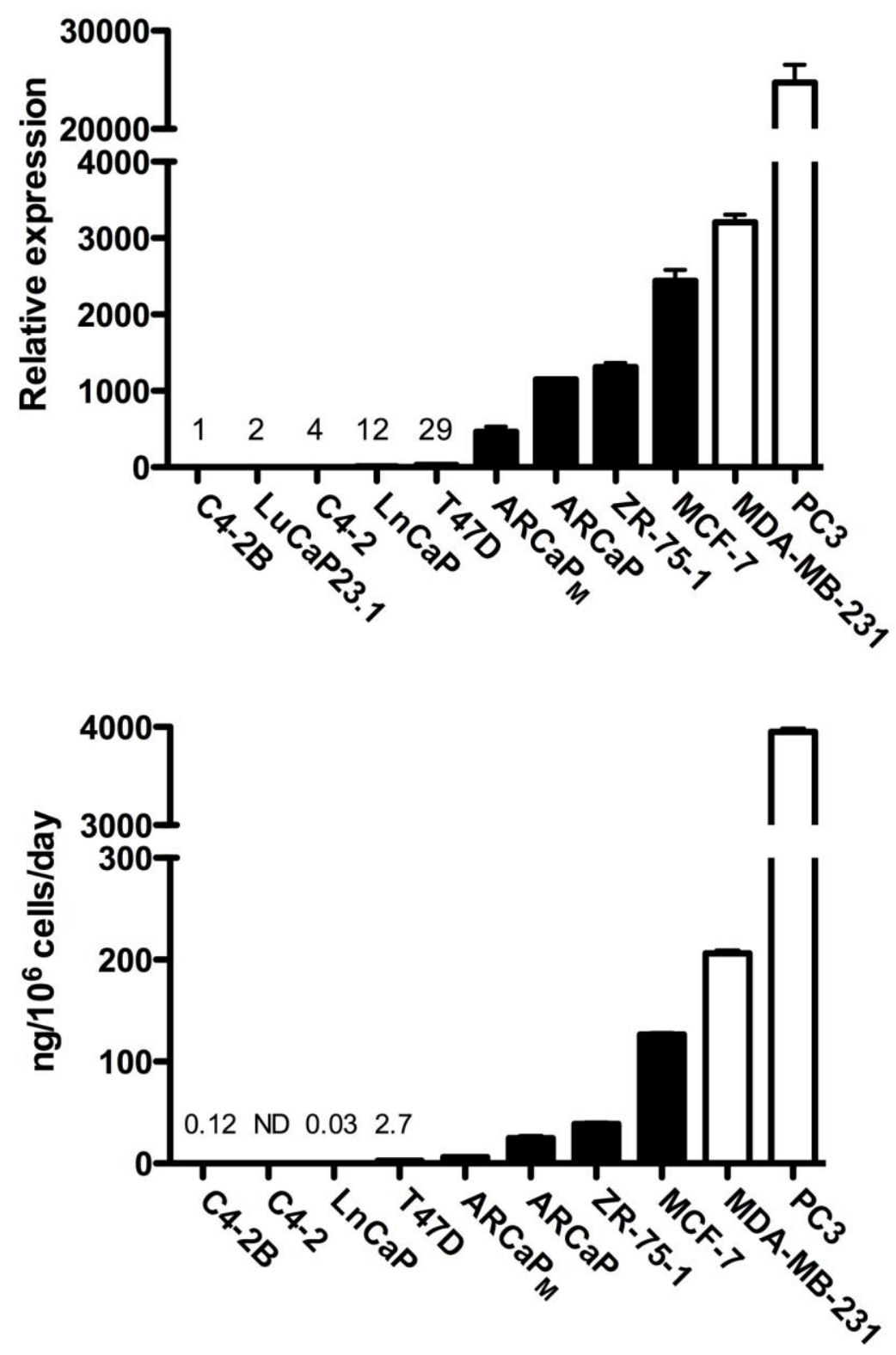

Figure 1 
Fig. 2

Click here to download line figure: Figure 2.ppt

\section{DKK1 Exon 1}

CpG island

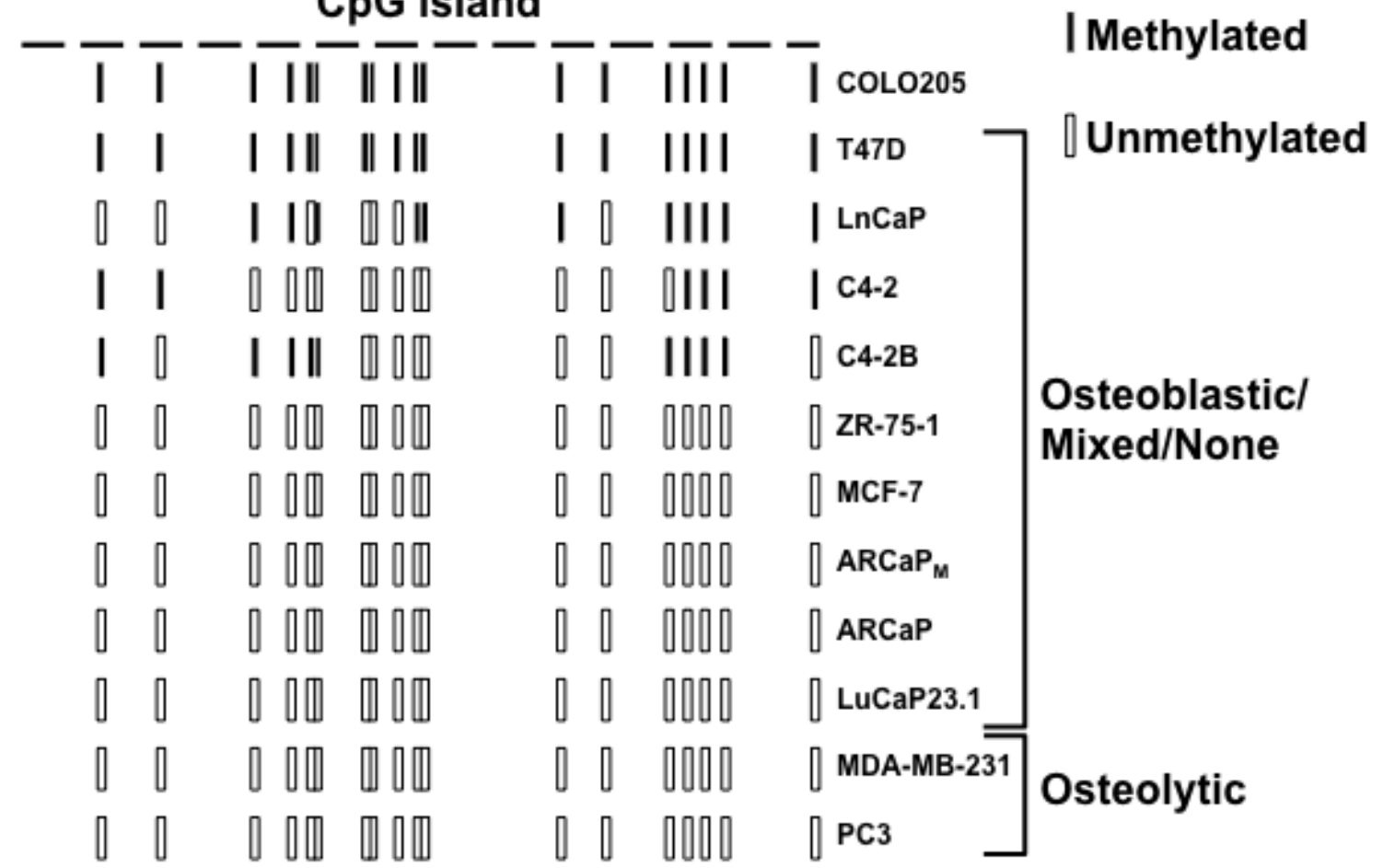

Figure 2 
Fig. 4B
Click here to download line figure: Figure 4B.ppt

Fig. 4B
Click here to download line figure: Figure 4B.ppt

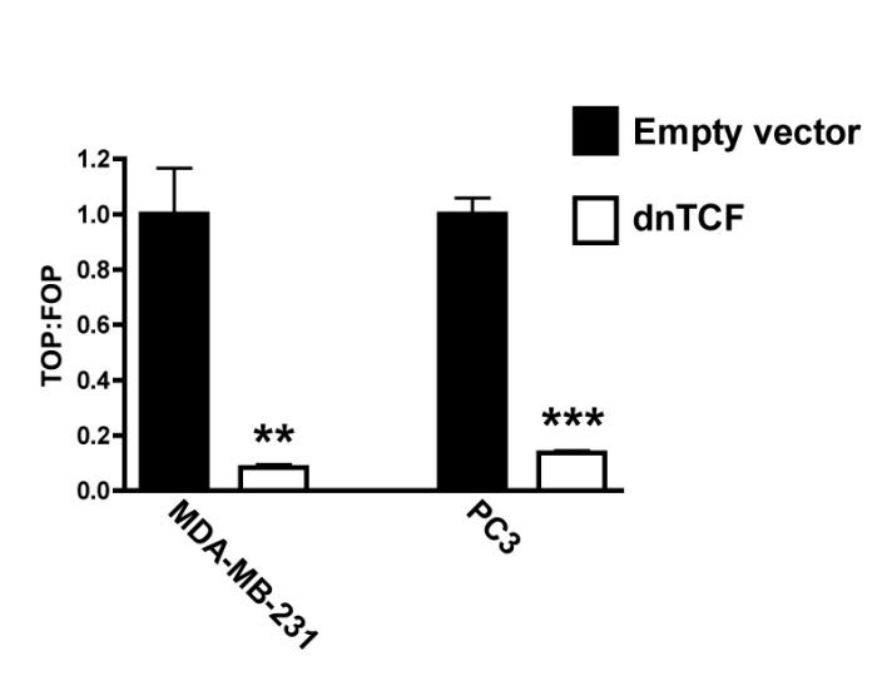

Figure 4B

(

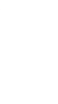


LRP5

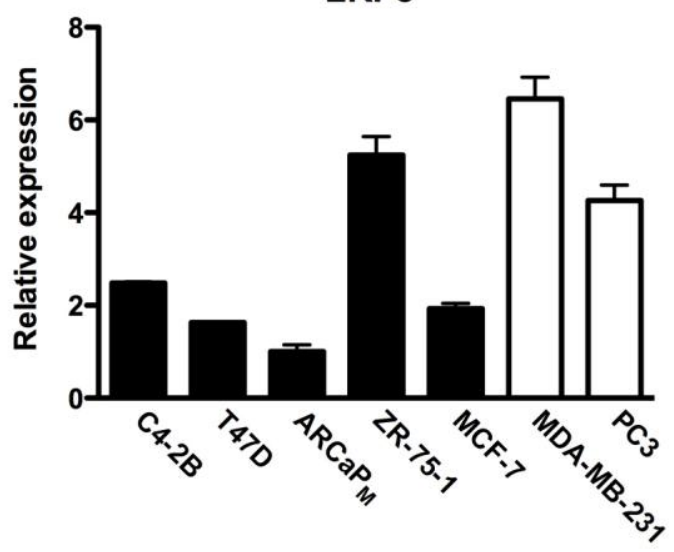

SOST

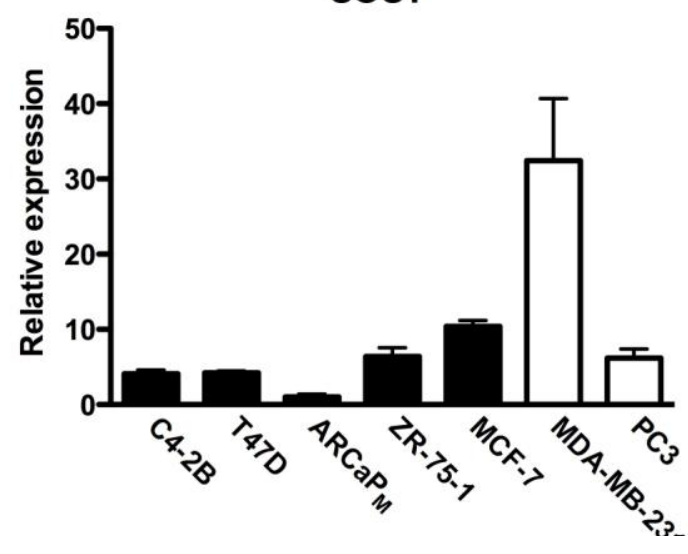

Krm1

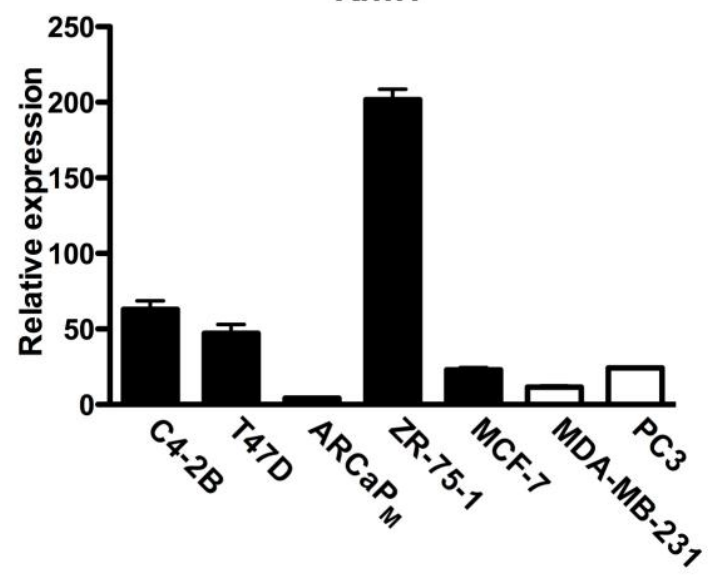

LRP6

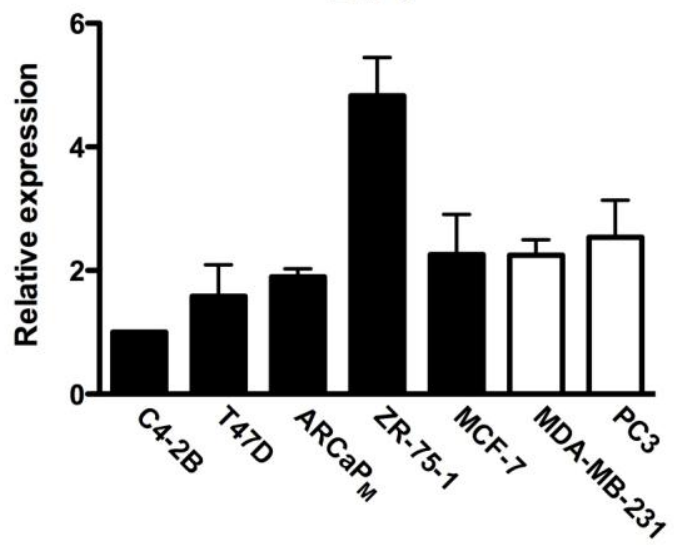

MESD

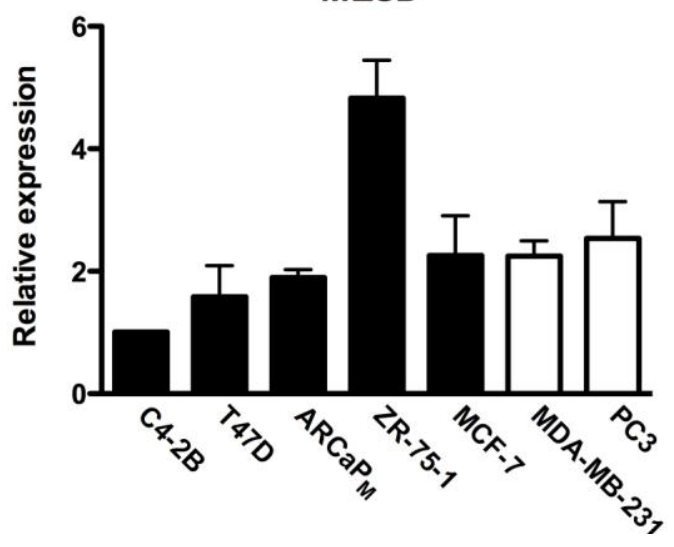

Krm2

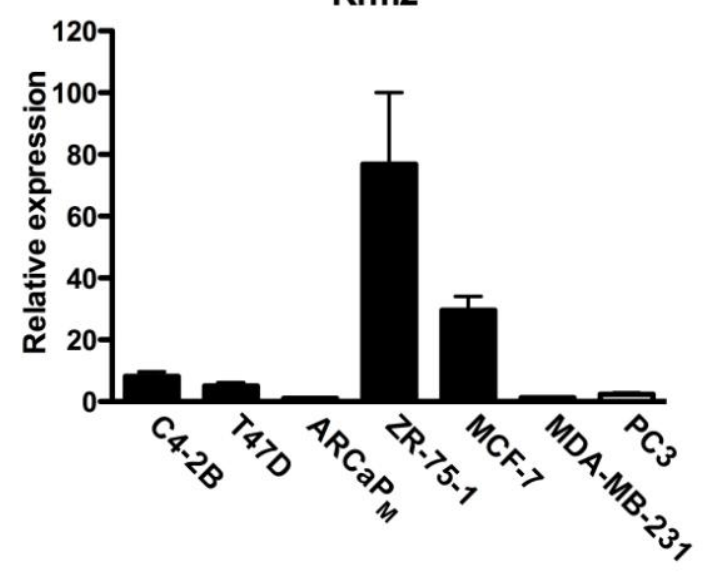

Figure 5 
Fig. 6
Click here to download line figure: Figure 6.ppt

Fig. 6
Click here to download line figure: Figure 6.ppt

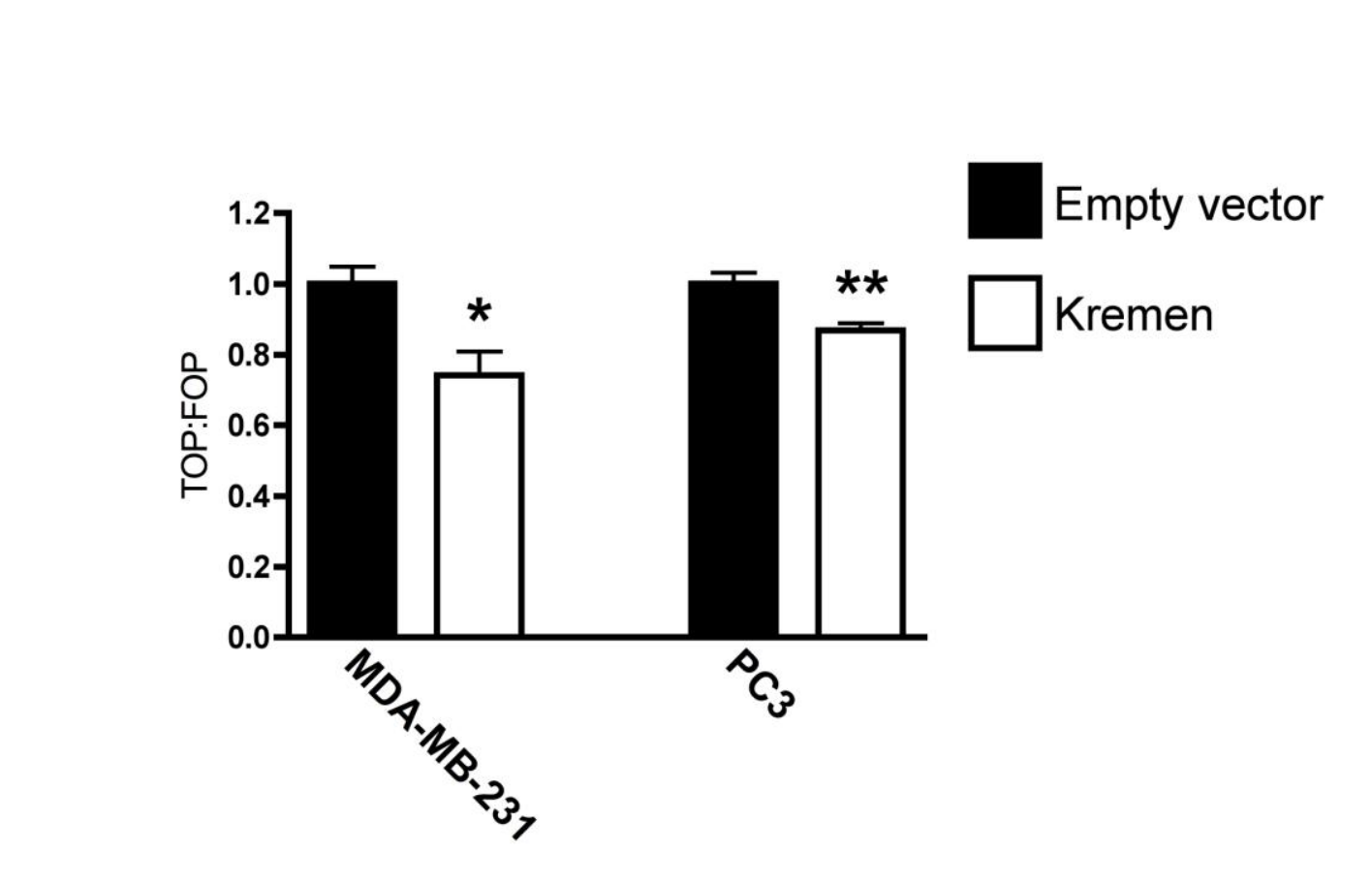

Figure 6

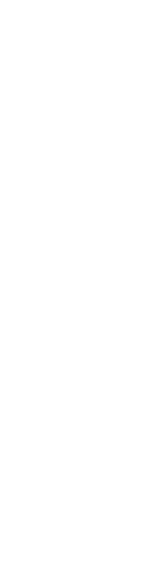

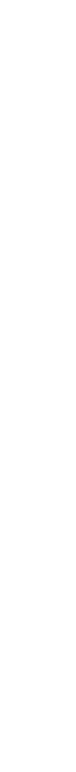

